\title{
Generalized Hartree-Fock theory for the dispersion relations of interacting fermionic lattice systems
}

\author{
Christina V. Kraus ${ }^{1,2}$ and Tobias J. Osborne ${ }^{3}$ \\ ${ }^{1}$ Institute for Quantum Optics and Quantum Information of the Austrian Academy of Sciences, A-6020 Innsbruck, Austria \\ ${ }^{2}$ Institute for Theoretical Physics, University of Innsbruck, A-6020 Innsbruck, Austria \\ ${ }^{3}$ Leibniz Universität Hannover, Institute of Theoretical Physics, Appelstrasse 2, D-30167 Hannover, Germany
}

(Received 5 June 2012; published 15 November 2013)

\begin{abstract}
We study the variational solution of generic interacting fermionic lattice systems using fermionic Gaussian states and show that the process of gaussification, leading to a nonlinear closed equation of motion for the covariance matrix, is locally optimal in time by relating it to the time-dependent variational principle. By linearizing our nonlinear equation of motion around the ground-state fixed point we describe a method to study low-lying excited states leading to a variational method to determine the dispersion relations of generic interacting fermionic lattice systems. This procedure is applied to study the attractive and repulsive Hubbard model on a two-dimensional lattice, as well as the stability of the Hofstadter butterfly structure in the presence of interactions.
\end{abstract}

DOI: 10.1103/PhysRevB.88.195126

PACS number(s): 05.30.Fk, 02.70.Hm, 05.10.-a

\section{INTRODUCTION}

The low-lying excited states of a quantum system are of central importance because they are, in contrast to the ground state, accessible in experiments. For example, spectral functions can be directly measured, revealing the particle content of a system. However, many theoretical models involve complicated many-body interactions, and an exact solution for their ground state and excitations are not known. In these cases, we must rely on numerical methods to find good approximations to the low-energy states of the system.

In the case of one-dimensional lattice systems, the density matrix renormalization group (DMRG) ${ }^{1,2}$ has emerged as the most successful numerical method. By understanding the DMRG as an application of the variational method to the matrix product state (MPS) ${ }^{3,4}$ class, many generalizations have been recently proposed. This growing collection of powerful algorithms now allows for the determination of the ground state, low-lying eigenstates, and time evolution of generic one-dimensional quantum spin systems. ${ }^{5,6}$

Comparatively less progress has been achieved in understanding the low-energy physics of fermionic systems; fermions are the building blocks of all matter, and are thus central to many exciting effects in condensed matter physics, including superconductivity, superfluidity, and the quantum Hall effect. While an MPS-based approach can be directly applied to one-dimensional fermionic systems, via the JordanWigner transformation, this is no longer possible for higherdimensional systems. Here, fermionic tensor networks, including fermionic projected entangled pair states (PEPS) ${ }^{7}$ and the multiscale entanglement renormalization ansatz (MERA) ${ }^{8}$ have been recently proposed for their study. ${ }^{9-11}$ However, so far, none of these approaches have been applied to describe the excitations of a fermionic quantum system.

Another possibility to find ground-state approximations of fermionic systems is to use other variational trial wave functions such as Slater determinants (Hartree-Fock theory) or Gutzwiller projected wave functions. These have also been used to describe excitations in the past, in the framework of time-dependent Hartree-Fock theory (see, e.g., Refs. 12 and 13) and time-dependent Gutzwiller theory. ${ }^{14}$ Recently, a technique based on the variational class of fermionic
Gaussian states (fGS) ${ }^{15}$ has been proposed. ${ }^{16}$ This method allows one to find approximate solutions for ground and thermal states, as well as the time evolution of interacting fermionic lattice systems in any dimension and geometry. An application to the two-dimensional (2D) spinfull FermiHubbard mode has established that these algorithms are stable and efficient, and provided results in agreement with, and going beyond, quantum Monte Carlo. The fGS class generalizes the basic building blocks of our understanding of fermionic matter, namely BCS states for superfluid phases, and Slater determinants (Hartree-Fock theory) for Mott and fermionic spin states to the framework of generalized Hartree-Fock theory (gHFT) ${ }^{17}$ which is a strict superset of Hartree-Fock theory. As fGS can be described using a number of variational parameters scaling polynomially in the system size, it allows the efficient simulation of large systems.

In this work, inspired by the utility of the fGS class for capturing a wide range of physically relevant phases, we take the next step and attempt to describe the low-lying excited states of interacting fermionic lattice systems. Hence we begin by introducing the general model of interacting fermions we investigate in the framework of gHFT. Then we apply the time-dependent variational principle (TDVP) $)^{12,18}$ to derive a locally (in time) optimal effective equation for the time evolution within the fGS variational class. An expansion of this equation around the ground-state solution thus leads to an approximation of the low-lying excited states. We demonstrate the power of our approach by investigating two examples: First, we discuss the two-dimensional Hubbard model on a $31 \times 31$ square lattice, both in the attractive and repulsive regimes, deriving the excitation spectrum, and discussing the nature of the excitations. Second, we apply our approach to a model exhibiting a Hofstadter butterfly structure and analyze the effect of interactions on a $6 \times 6$ lattice.

\section{EXCITATION SPECTRA WITHIN GENERALIZED HARTREE-FOCK THEORY}

In the following section we explain how we can obtain an approximation to the excitation spectra within gHFT. We start with defining the problem we want to solve and give a brief 
introduction into gHFT. Then we show how we can use the TDVP to achieve our goal.

\section{A. Interacting fermionic lattice systems within gHFT: A review}

Throughout this work we consider a system of $M$ fermionic modes localized on a lattice in $d$ dimensions. This is described by fermionic creation and annihilation operators obeying the canonical anticommutation relations (CARs) $\left\{a_{k}^{\dagger}, a_{l}\right\}=\delta_{k, l}$, $\left\{a_{k}, a_{l}\right\}=0$. Such a system can be equivalently described by $2 M$ real Majorana operators $c_{j}=a_{j}^{\dagger}+a_{j}$ and $c_{j+M}=$ $(-i)\left(a_{j}^{\dagger}-a_{j}\right)$ obeying the CAR $\left\{c_{j}, c_{k}\right\}=2 \delta_{j k}$. Many systems of importance in condensed-matter physics are modeled by Hamiltonians involving two-body interactions. In the Majorana language, the most general such model is written as

$$
H=i \sum_{k l} T_{k l} c_{k} c_{l}+\sum_{k l m n} U_{k l m n} c_{k} c_{l} c_{m} c_{n},
$$

where $T=-T^{T} \in \mathbb{R}^{2 M \times 2 M}$ and $U_{k l m n} \in \mathbb{R}$ is antisymmetric under the exchange of any two adjacent indices.

In the following we aim at finding an approximation to the excitation spectra based on some appropriate class of variational trial states. As pointed out in the Introduction, the fermionic Gaussian states (fGS) might be a suitable candidate since they allow, among others, to describe superconducting and spin-ordered phases. Note that the use of fGS as a class of variational states is also known as generalized Hartree-Fock theory (gHFT). The fGS class is defined as the set of all states which are exponentials of a quadratic form in the fermionic operators. That is we can write the density operator of the system as $\rho \sim \exp \left[-c^{T} K c\right]$, where $K^{T}=-K \in \mathbb{R}^{2 M \times 2 M}$ and $c=\left(c_{1}, \ldots, c_{2 M}\right)$. Such states have the important property that they fulfill Wick's theorem, and thus can be completely described on a single-particle level via the real and antisymmetric covariance matrix (CM) $\Gamma_{k l}=i / 2\left\langle\left[c_{k}, c_{l}\right]\right\rangle$ (see, e.g., Ref. 15). For physical states the $\mathrm{CM}$ obeys the inequality $i \Gamma \leqslant \mathbb{1}$, while for pure states we have $\Gamma^{2}=-\mathbb{1}$. Note that every pure fGS is the ground state of a quadratic Hamiltonian $H=i \sum_{k l} h_{k l} c_{k} c_{l}$, where $h=-h^{T} \in \mathbb{R}$. Furthermore, Gaussian states remain Gaussian under time evolution according to any quadratic Hamiltonian, so that the time evolution can be formulated in terms of the CM alone as $\dot{\Gamma}(t)=4[h, \Gamma(t)]$.

Recently, in Ref. 16, the fGS variational class has been used to find approximate solutions for the ground and thermal states of (1). Since it will become important later on, we briefly review how to approximate the time evolution of an interacting system of the form (1) using fGS. Since $H$ generically includes nonquadratic interactions, any infinitesimal time step $\Delta t$ takes us out of the fGS class. Thus, we have to project back into the set of fGS after each time step. In Ref. 16 this was done via the process of gaussification, where Wick's theorem is invoked after each time step to re-express the system's state as an fGS. In this way, a closed evolution of the CM could be derived:

$$
\begin{gathered}
\dot{\Gamma}(t)=4\left[h^{(6)}(\Gamma(t)), \Gamma(t)\right], \\
h^{(6)}(\Gamma)=T+6 \operatorname{tr}_{2}[U \Gamma] .
\end{gathered}
$$

Thus time evolution can be formulated as the nonlinear evolution according to a quadratic but state-dependent Hamiltonian. In the following we use the time-dependent variational principle to prove that gaussification, used ad hoc in Ref. 16, is actually locally optimal in time within the set of fGS. But before that, let us briefly mention that Eq. (2) allows us to determine the ground state covariance matrix $\Gamma_{0}$ via an imaginary time evolution with respect to the quadratic, but state dependent, Hamiltonian $H=$ $i \sum_{k, l} h^{(6)} c_{k} c_{l}$ (Ref. 16): Starting from an arbitrary pure state $\Gamma(0)$ the evolution $\Gamma(t)=O(t) \Gamma(0) O(t)^{T}$, where $O(t)=$ $\mathcal{T} \exp \left\{\int_{0}^{t} d t^{\prime} 2\left[\Gamma\left(t^{\prime}\right), h^{(6)}\left(\Gamma\left(t^{\prime}\right)\right)\right]\right\}$, leads to $\Gamma_{0}$. Note that such an approach scales only polynomially in the system size and can be applied to any dimension and geometry.

\section{B. Equivalence of gaussification and the TDVP}

The goal of this subsection is to show the equivalence of gaussification and the TDVP. To this end, we briefly recall the TDVP: Suppose we have some variational class of states $\left\{|\psi(\mathbf{x})\rangle \mid \mathbf{x} \in \mathbb{R}^{D}\right\}$. We aim to solve the time-dependent Schrödinger equation $d / d t|\psi(\mathbf{x})\rangle=-i H|\psi(\mathbf{x})\rangle$ as best as possible while remaining in our class. Unfortunately, in general, the vector $i H|\psi(\mathbf{x})\rangle$ is not an element of the tangent space, and we can only find an approximate solution. The optimal way to do this is to carry out the minimization $\min _{\dot{\mathbf{x}}} \| \dot{x}^{j} \partial_{j}|\psi(\mathbf{x})\rangle+i H|\psi(\mathbf{x})\rangle \|$, where we have written $|\psi(\mathbf{x})\rangle$ as a linear combination of vectors in the tangent space, and $\partial_{j}=\partial / \partial x_{j}$. If we perform this optimization at each time step, and take the limit of infinitesimal step size, we arrive at the locally optimal evolution within our variational class. Note that this minimization is equivalent to solving for the Euler-Lagrange equation $\frac{d}{d t} \frac{\partial \mathcal{L}}{\partial \dot{x}_{j}}-\frac{\partial \mathcal{L}}{\partial x_{j}}=0$ with Lagrangian $\mathcal{L}=\left\langle\psi(\mathbf{x})\left|\left(-i \frac{d}{d t}-H\right)\right| \psi(\mathbf{x})\right\rangle$.

We now show that gaussification is locally optimal by demonstrating its equivalence to the TDVP. To see this, note that any pure fermionic Gaussian state can be represented as

$$
\left|\psi_{G}(t)\right\rangle=\mathcal{T} \exp \left[\frac{i}{2} \int_{0}^{t} \sum_{k, l} G_{k l}\left(t^{\prime}\right) c_{k} c_{l}\right]|0\rangle,
$$

where $G=-G^{T}$ is a real matrix. Thus $\Gamma$ and $G$ are related via $\dot{\Gamma}=[G, \Gamma]$. This relation is imposed in a Lagrangian using a Lagrange multiplier $\Lambda$, and we obtain for the TDVP Lagrangian the expression

$$
\begin{aligned}
\mathcal{L} & =\left\langle\psi(\mathbf{x})\left|\left(-i \frac{d}{d t}-H\right)\right| \psi(\mathbf{x})\right\rangle \\
& =\operatorname{tr}\left\{\Gamma\left[G+h^{(3)}(\Gamma)\right]\right\}+\operatorname{tr}\{\Lambda(\dot{\Gamma}-[G, \Gamma])\},
\end{aligned}
$$

where $h^{(3)}(\Gamma)=T+3 \operatorname{tr}_{2}[U \Gamma]$. The Euler-Lagrange equations of motion immediately lead to $\dot{\Gamma}=4\left[h^{(6)}, \Gamma\right]$, which are exactly those arising from gaussification in Ref. 16. Thus we obtain a locally optimal smooth path in our variational manifold, as depicted in Fig. 1.

\section{Excitation spectra in gHFT}

Now we use the optimality of the TDVP to derive an approximation of the excitation spectrum within the fGS variational class. To this end we linearize our TDVP equation of motion around the variational ground state given by $\Gamma_{0}$, i.e., we write $\Gamma(t)=\Gamma_{0}+\epsilon \Gamma_{1}+O\left(\epsilon^{2}\right)$, with real $\Gamma_{1}=-\Gamma_{1}^{T}$. In order to obtain the excitation spectrum, we 


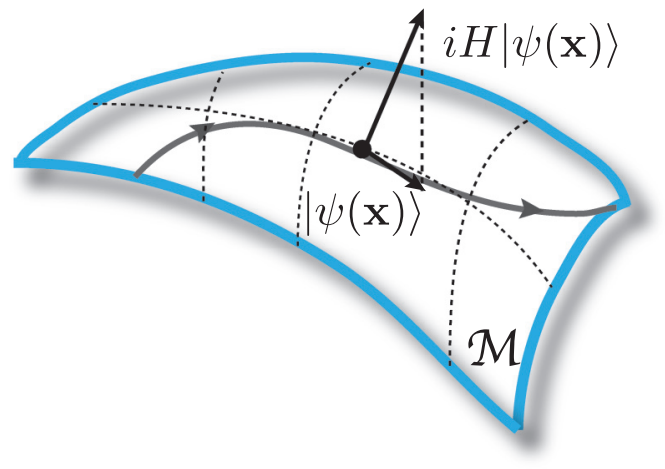

FIG. 1. (Color online) Graphical representation of the TDVP within the variational manifold $\mathcal{M}$ of Gaussian states represented by state vectors $|\psi(\mathbf{x})\rangle$. The time evolved vector $i H|\psi(\mathbf{x})\rangle$ is in general not an element of the manifold $\mathcal{M}$, and we have to find an optimal approximation within the variational manifold. Thus, we obtain the optimal path $|\psi(\mathbf{x})\rangle$ within the manifold $\mathcal{M}$.

write the wave function of the excited state as an expansion around the ground state $\left|\Psi_{0}(t)\right\rangle:|\Psi(t)\rangle=\left|\Psi_{0}(t)\right\rangle+$ $\left|\Psi_{1}(t)\right\rangle$. We express $\left|\Psi_{1}(t)\right\rangle=\sum_{n>0} \gamma_{n}(t) e^{i E_{n} t / \hbar}|n\rangle$, where $|n\rangle$ are the eigenfunctions of the Hamiltonian with energies $E_{n}$. Then, it follows immediately that $\left[\Gamma_{1}(t)\right]_{k l}=$ $\sum_{n, m} \gamma_{n}(t) \gamma_{m}(t)^{*} e^{i\left(E_{n}-E_{m}\right) t / \hbar}\left\langle m\left|i c_{k} c_{l}\right| n\right\rangle$. Thus, if we write $\Gamma_{1}(t)=e^{i \omega t} \hat{\Gamma}(\omega)$ (note that we have to take the real part of this ansatz when calculating physical properties since the CM is always real) we arrive at the following eigenvalue equation, which is the main result of this work:

$$
i \omega \hat{\Gamma}_{1}(\omega)=\left[h^{(6)}\left(\Gamma_{0}\right), \hat{\Gamma}_{1}(\omega)\right]+6\left\{\operatorname{tr}\left[U \hat{\Gamma}_{1}(\omega)\right], \Gamma_{0}\right\},
$$

and we obtain the solutions $\omega_{n, m}=E_{n} \pm E_{m}$. It is easy to show that in the noninteracting case where $U=0$, the above equation leads to the exact results in the free case, i.e., we obtain the eigenvalues of $T$, cf. Eq. (1).

While (5) gives the general solution for the excitation spectrum within the fGS variational class, this matrix equation is, in general, hard to solve. The problem may be simplified by rewriting it as an eigenvalue equation via vectorization:

$$
\begin{aligned}
i \omega\left|\Gamma_{1}\right\rangle & =\mathcal{V}\left|\Gamma_{1}\right\rangle, \\
\mathcal{V} & =h^{(6)} \otimes \mathbb{1}+\mathbb{1} \otimes h^{(6)}-6(\Gamma \otimes \mathbb{1}+\mathbb{1} \otimes \Gamma) U,
\end{aligned}
$$

where $\mathcal{V}$ is a $4 M^{2} \times 4 M^{2}$ matrix. Thus, the problem reduces to diagonalizing the matrix $\mathcal{V}$, which can be numerically demanding for large systems. The problem is easier in the presence of additional symmetries; however, in the case of a translation invariant system the problem simplifies considerably. In this case the matrix $\Gamma_{1}$ is block diagonal in Fourier space, i.e., $\hat{\Gamma}_{1}=\bigoplus_{\mathbf{k}} \Gamma_{1}(\mathbf{k})$, with modes $\mathbf{k}$ and $-\mathbf{k}$ paired. Thus, for each momentum mode $\mathbf{k}$, we only need to solve the reduced equation $i \omega(\mathbf{k})\left|\Gamma_{1}(\mathbf{k})\right\rangle=\mathcal{V}(\mathbf{k})\left|\Gamma_{1}(\mathbf{k})\right\rangle$. In this way we can extract the dispersion relation $\omega(\mathbf{k})$. Furthermore, since the CM contains all information about a fGS, we can also infer the nature of the excitations by looking at $\Gamma_{1}(\mathbf{k})$.

\section{NUMERICAL APPLICATION}

To illustrate the power of our approach, we apply it now to two examples, namely the two-dimensional Hubbard model and to the Hoftstadter butterfly model in the presence of interactions, both on a square lattice. We would like to stress that we are mainly interested in showing that our approach allows for an efficient numerical implementation and that furthermore, the results obtained in this way are in qualitative agreement with results obtained by other known methods. A detailed comparison with known techniques is left as future work.

\section{A. Example I: The 2D Hubbard model on a square lattice}

The Hubbard model describes an interacting fermionic lattice system of particles with two internal spin states $\sigma=$ $\uparrow, \downarrow$,

$$
H_{\mathrm{Hubb}}=-t \sum_{\langle\mathbf{x}, \mathbf{y}\rangle, \sigma} a_{\mathbf{x}, \sigma}^{\dagger} a_{\mathbf{y}, \sigma}+u \sum_{\mathbf{x}} n_{\mathbf{x} \uparrow} n_{\mathbf{x} \downarrow}-\mu \sum_{\mathbf{x}, \sigma} n_{\mathbf{x} \sigma},
$$

where $\mathbf{x}$ denotes a position on the lattice, $\langle\cdots\rangle$ indicates a summation over nearest neighbors, and $n_{\mathbf{x} \sigma}=a_{\mathbf{x} \sigma}^{\dagger} a_{\mathbf{x} \sigma}$ is a particle number operator. We take the hopping parameter $t$ to be real and consider $u$ positive (negative) in case of a repulsive (attractive) interaction. The chemical potential $\mu$ fixes the filling of the lattice.

Despite its simple structure the Hubbard model allows for a wide range of physical phases, including Mott and spin-ordered phases in the case of a repulsive interaction, and superfluid phases in case of an attractive interaction. It is even believed that the doped Hubbard model at positive $u$ may provide a description of high-temperature superconductivity. However, unless we consider very special parameter regimes, an exact solution of the model is unknown, and despite an intense theoretical and numerical effort the precise structure of its phase diagram remains an open question. Since the literature on the Hubbard model is vast, we do not aim at carrying out a detailed comparison with the literature, but rather focus on presenting that gHFT allows us to capture some of the known qualitative results.

In the following we determine the excitation spectrum of the translation-invariant Hubbard model with periodic boundary conditions (PBCs) in two dimensions within the fGS variational class using Eq. (5). To calculate $\mathcal{V}$ we need the $\mathrm{CM}$ of the ground state $\Gamma_{0}$. For the translation invariant case with PBCs it has been shown in Ref. 17 that $\Gamma_{0}$ can be obtained via a two-parameter optimization for arbitrary filling, both in the attractive and repulsive regimes. We use this result to numerically determine $\Gamma_{0}$ on a $31 \times 31$ lattice, transform into Fourier space, and are left, for each $\mathbf{k}$, with the diagonalization of an $64 \times 64$ matrix, leading to the dispersion $\omega_{n, m}(k)$.

In order to learn more about the nature of the excitations, we consider order parameters that characterize the pairing and the spin of the state. We do not aim at an exhaustive analysis but rather introduce simple quantities that give some qualitative insight into the structure of the state. Following Ref. 19, we define for each $\mathbf{k}, \Delta(\mathbf{k})=\sum_{\sigma, \sigma^{\prime}=\uparrow, \downarrow}\left|\left\langle a_{ \pm \mathbf{k} \sigma}^{\dagger} a_{ \pm \mathbf{k} \sigma}^{\dagger}\right\rangle\right|^{2}$. This parameter characterizes the strength of the pairing between fermions with momentum $\pm \mathbf{k} . \Delta(\mathbf{k})$ is optimal, for example, for the state $\left|\Psi_{p}\right\rangle=\frac{1}{\sqrt{2}}\left(\mathbb{1}+a_{\mathbf{k} \uparrow}^{\dagger} a_{-\mathbf{k} \downarrow}^{\dagger}\right)|0\rangle$. To get some insight into the spin properties of the system, we consider two 
order parameters. The first one $S_{z}(\mathbf{k})=\left\langle n_{\mathbf{k} \uparrow}-n_{\mathbf{k} \downarrow}\right\rangle$ indicates if there is a nonvanishing total spin associated with the momentum mode k. $S_{z}(\mathbf{k})$ achieves its maximum (minimum), for example, for the state $\left|\Psi_{z}^{+}\right\rangle=a_{\mathbf{k} \uparrow}^{\dagger}|0\rangle\left(\left|\Psi_{z}^{-}\right\rangle=a_{\mathbf{k} \downarrow}^{\dagger}|0\rangle\right)$. The second one $S_{s}=\left|\left\langle a_{\mathbf{k} \uparrow}^{\dagger} a_{-\mathbf{k} \downarrow}\right\rangle\right|^{2}+\left|\left\langle a_{-\mathbf{k} \uparrow}^{\dagger} a_{\mathbf{k} \downarrow}\right\rangle\right|^{2}$ achieves its maximum, for example, for states of spin-singlet form $\left|\Psi_{s}\right\rangle=\frac{1}{\sqrt{2}}\left(a_{\mathbf{k} \uparrow}^{\dagger} \pm a_{-\mathbf{k} \downarrow}^{\dagger}\right)|0\rangle$.

\section{B. Attractive Hubbard model}

Let us discuss our results for the Hubbard model in the attractive case for two exemplary sets of parameters $(u, \mu)=$ $(-4,1),(-2,2)$. We discuss first some properties of the ground states. We have calculated the filling $n=N /\left(2 \cdot 31^{2}\right)$ and the pairing per particle ${ }^{19} p=\sum_{i, j, \sigma, \sigma^{\prime}}\left|\left\langle a_{i \sigma}^{\dagger} a_{j \sigma^{\prime}}^{\dagger}\right\rangle\right|^{2} / N$, where $N$ is the number of particles in the lattice. For $(u, \mu)=(-4,1)$ we get $n=0.83, p=0.028$ and for $(u, \mu)=(-2,2)$ we obtain $n=0.24, p=0.45$. We consider configurations far from halffilling since at half-filling the Hubbard model has additional symmetries leading to a highly degenerate ground state which severely complicates analysis of the excitations. The attractive Hubbard model supports superfluid phases indicated by a nonvanishing pairing per particle. Thus, the ground state is gapped for the two sets of parameters.

Next, we investigate the excitation spectra. As has been shown Ref. 20 using quantum Monte Carlo techniques, the system behaves qualitatively rather close to what is expected from BCS theory which is a subclass of gHFT. Thus, a band structure similar to BCS theory with two bands $E_{k}^{>}>0$ and $E_{k}^{<}=-E_{k}^{>}$is expected. This is confirmed by our results that are depicted in Fig. 2. Note that we plot only the dispersion with $E_{k}^{>}>0$. In both cases, the excitation spectra are sixfold degenerate, but the difference of the eigenvalues lies within the numerical errors, and hence these excitations cannot be distinguished. Thus, in order to obtain the values of the order parameters $\Delta, S_{z}, S_{s}$, we consider a $\mathrm{CM}$ which is an equal weight statistical mixture of these six solutions. We find that in the case of $u=-4, \mu=1$ the order parameters $S_{S}\left(k_{x}, 0\right)$ have a flat dispersion along $k_{x}$. The results for the other order parameters are shown in Fig. 3. Note that the results are symmetric around $k_{x}=\pi$, and thus we have only plotted half of the Brillouin zone.

\section{Repulsive Hubbard model}

Now we discuss excitations in the repulsive Hubbard model for $(u, \mu)=(4,0)$ and $(u, \mu)=(4,-3)$, where the fillings are $n=0.18$, and $n=0.81$, respectively. Here, as predicted in Ref. 17, the ground state is never paired, i.e., $p=0$. Spinordered phases within a gHFT treatment are only predicted for
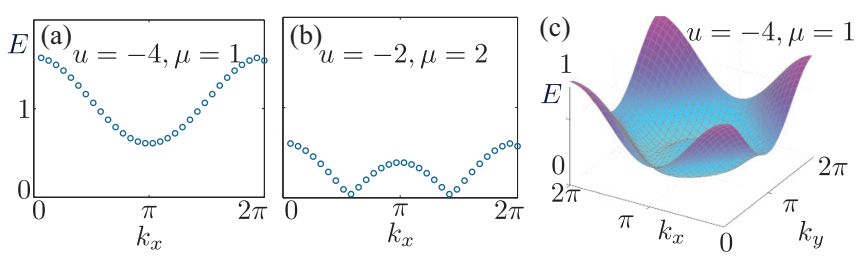

FIG. 2. (Color online) Dispersion relation for the Hubbard model on a $31 \times 31$ lattice for attractive interactions.
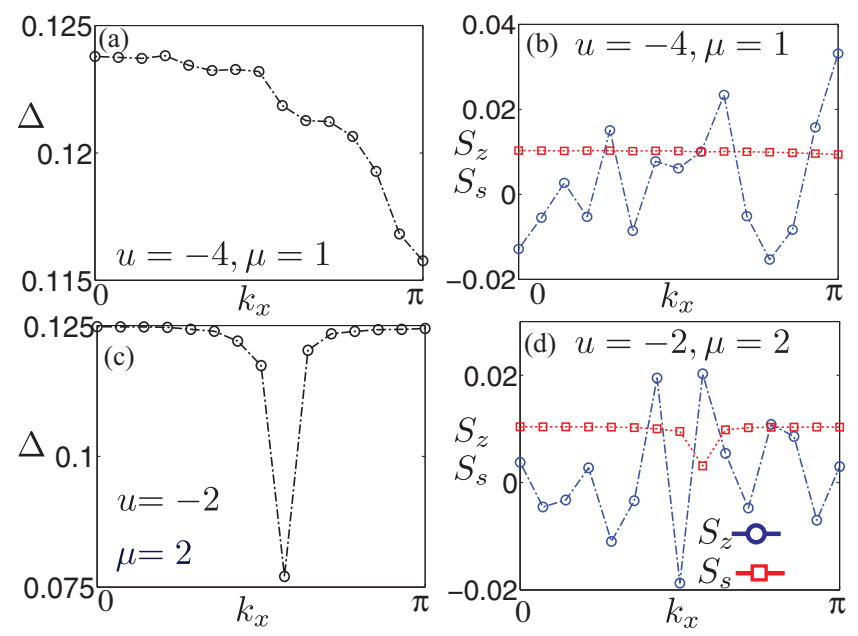

FIG. 3. (Color online) Order parameters $\Delta(\mathbf{k})=$ $\sum_{\sigma, \sigma^{\prime}=\uparrow, \downarrow}\left|\left\langle a_{ \pm \mathbf{k} \sigma}^{\dagger} a_{ \pm \mathbf{k} \sigma}^{\dagger}\right\rangle\right|^{2}, \quad S_{z}(\mathbf{k})=\left\langle n_{\mathbf{k} \uparrow}-n_{\mathbf{k} \downarrow}\right\rangle \quad S_{s}=\left|\left\langle a_{\mathbf{k} \uparrow}^{\dagger} a_{-\mathbf{k} \downarrow}\right\rangle\right|^{2}+$ $\left|\left\langle a_{-\mathbf{k} \uparrow}^{\dagger} a_{\mathbf{k} \downarrow}\right\rangle\right|^{2}$ on a $31 \times 31$ lattice for attractive interactions. (a) and (b) are for $(u, \mu)=(-4,1)$, while (c) and (d) are for $(u, \mu)=(-2,2)$.

half-filling, or large $u$. The excitation spectrum is depicted in Fig. 4. We find again that the excitation spectrum is sixfold degenerate, but the degeneracy is within the numerical errors of our approach. Furthermore, we find two branches with energies $E_{k}^{>}=-E_{k}^{<}$and again plot only $E_{k}^{>}$. In Fig. 4(c) we compare the dispersion for $u=4$ at the two different fillings. We find that the qualitative behavior of the dispersion changes with the filling. This is very similar to the results obtained in the limit of large $U$ in Ref. 21, but with the role of large and small doping interchanged. This is no contradiction, since the two methods work in very different regimes, and we expect that gHFT works best close to the noninteracting case. Finally, we study the nature of our excitations. Again, we take an equal weight statistical mixture of these six solutions to calculate the order parameters, and find that $S_{z}=0$, while the dispersion for the other two order parameters is flat, $S_{s}(\mathbf{k}) \approx 0.01$ and $\Delta(\mathbf{k}) \approx$ 0.13 . Note that while gHFT always leads to an unpaired ground state for the repulsive Hubbard model, it is able to capture a paired excited state.

\section{Example II: The Hofstadter butterfly}

As a second example, we study the Hofstadter butterfly in the presence of attractive interactions on an $N \times N$ square
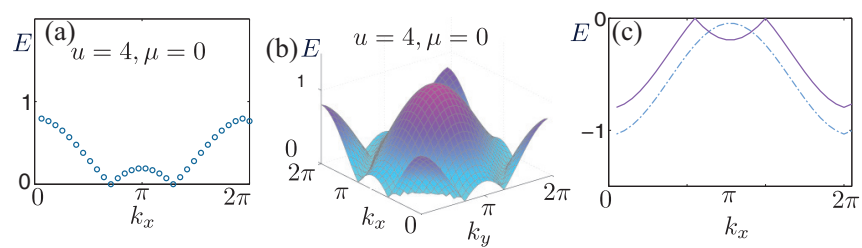

FIG. 4. (Color online) Dispersion relation for the Hubbard model on a $31 \times 31$ lattice for repulsive interactions. (c) Comparison of the dispersion for $u=4$ and $\mu=-3$ (dashed, $n=0.7$ ) and $\mu=0$ (solid, $n=0.18$ ). 

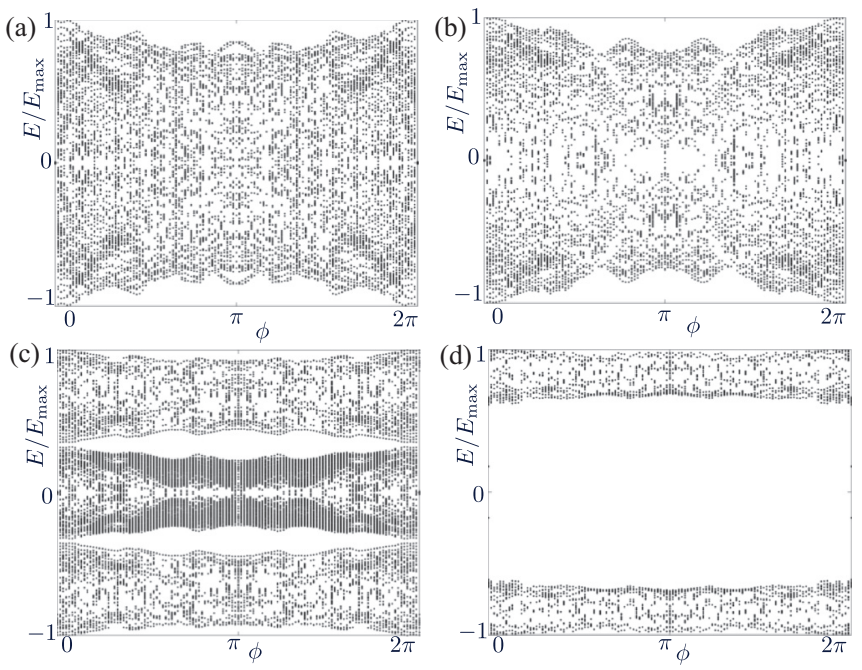

FIG. 5. Hofstadter butterfly in the presence of interactions [Eq. (8)] on a $6 \times 6$ lattice. Starting from the free case $u=0$, where we recover the same butterfly structure as in Ref. 23 we increase the interaction to the values $u=-0.5$ (b), $u=-2$ (c), and $u=-4$ (d). While the butterfly structure is stable against interactions for small $u$ (b), we see that stronger interactions gap out and destroy the structure (c) and (d).

lattice. This is modeled with the Hamiltonian

$$
H_{B}=\sum_{\left\langle\mathbf{x}_{1}, \mathbf{x}_{2}\right\rangle} a_{\mathbf{x}_{1}}^{\dagger} a_{\mathbf{x}_{2}} e^{i \theta_{\mathbf{x}_{1}, \mathbf{x}_{2}}}+\text { H.c. }+u \sum_{\left\langle\mathbf{x}_{1}, \mathbf{x}_{2}\right\rangle} n_{\mathbf{x}_{1}} n_{\mathbf{x}_{2}} .
$$

Here $\theta_{\mathbf{x}_{1}, \mathbf{x}_{2}}$ is chosen to have the nontrivial value $\theta_{(x, y),(x+1, y)}=$ $x \phi$, with $0 \leqslant \phi \leqslant 2 \pi$ only in the $x$ direction, and $u<0$. For the case $u=0$ and $N \rightarrow \infty$, the excitation spectrum, if plotted against the angle $\phi$, results in the well-known butterflylike structure. ${ }^{22}$ For finite lattices, this structure is less pronounced, but still visible, as shown in Ref. 23. We take these results as a starting point to investigate the effects of an additional attractive interaction on the excitation spectrum on the Hofstadter model [Eq. (8)]. The results for a $6 \times 6$ lattice are shown in Fig. 5. For convenience we have normalized the energies such that the largest eigenvalues has the magnitude 1 , i.e., we depict $E / E_{\max }$. Starting from the free case $u=0$, where we obtain similar results as shown in Ref. 23 [see Fig. 5(a)], we increase the interaction strength. While the butterfly structure is stable against small interactions [Fig. 5(b)], it disappears for large $u$ where it is gapped out by bound states [Figs. 5(c) and 5(d)].

\section{CONCLUSION}

In summary, we have demonstrated that the TDVP applied to the set of fermionic Gaussian states allows for a variational approach to determine dispersion relations and the nature of the excitations in interacting fermionic systems. For translation invariant systems, the arising equations scale linearly in the system size, and can thus be applied to large systems in more than one dimension. As an illustrative example, we applied our approach to the the 2D Hubbard model in both the attractive and the repulsive regimes on a $31 \times 31$ lattice. The excitation spectra can be calculated efficiently, and we expect them to approximate well the physics of interacting fermionic lattice systems at least in the weakly interacting regime. Our results showed behavior that is in agreement with results obtained via other techniques. Furthermore, we have demonstrated the power of our approach by studying the stability of the Hofstadter butterfly in the presence of interactions on a square lattice.

\section{ACKNOWLEDGMENTS}

We thank I. Cirac, J. Haegeman, and F. Verstraete for useful discussion. This work was supported, in part, by the cluster of excellence EXC 201 Quantum Engineering and Space-Time Research, by the Deutsche Forschungsgemeinschaft (DFG), the EU grant QFTCMPS, and by the Austrian Ministry of Science BMWF as part of the UniInfrastrukturprogramm of the Research Platform Scientific Computing at the University of Innsbruck.
${ }^{1}$ S. R. White, Phys. Rev. Lett. 69, 2863 (1992).

${ }^{2}$ U. Schollwöck, Rev. Mod. Phys. 77, 259 (2005).

${ }^{3}$ F. Verstraete, V. Murg, and J. Cirac, Adv. Phys. 57, 143 (2008).

${ }^{4}$ J. I. Cirac and F. Verstraete, J. Phys. A 42, 504004 (2009).

${ }^{5}$ J. Haegeman, J. I. Cirac, T. J. Osborne, I. Pizorn, H. Verschelde, and F. Verstraete, Phys. Rev. Lett. 107, 070601 (2011).

${ }^{6}$ J. Haegeman, B. Pirvu, D. J. Weir, J. I. Cirac, T. J. Osborne,

H. Verschelde, and F. Verstraete, Phys. Rev. B 85, 100408 (2012).

${ }^{7}$ C. V. Kraus, N. Schuch, F. Verstraete, and J. I. Cirac, Phys. Rev. A 81, 052338 (2010).

${ }^{8}$ P. Corboz and G. Vidal, Phys. Rev. B 80, 165129 (2009).

${ }^{9}$ P. Corboz, G. Evenbly, F. Verstraete, and G. Vidal, Phys. Rev. A 81, 010303(R) (2010).

${ }^{10}$ C. Pineda, T. Barthel, and J. Eisert, Phys. Rev. A 81, 050303(R) (2010).

${ }^{11}$ T. Barthel, C. Pineda, and J. Eisert, Phys. Rev. A 80, 042333 (2009)
${ }^{12}$ P. M. A. Dirac, Proc. Cambridge Philos. Soc. 30, 376 (1930).

${ }^{13} \mathrm{G}$. Arrighini and C. Guidotti, Mol. Phys. 24, 631 (1972).

${ }^{14} \mathrm{G}$. Seibold and J. Lorenzana, Phys. Rev. Lett. 86, 2605 (2001).

${ }^{15}$ S. Bravyi, Quant. Inf. Comput. 5, 216 (2005).

${ }^{16}$ C. V. Kraus and J. I. Cirac, New J. Phys. 12, 113004 (2010).

${ }^{17}$ V. Bach, E. H. Lieb, and J. P. Solovej, J. Stat. Phys. 76, 3 (1994).

${ }^{18}$ P. W. Langhoff, S. T. Epstein, and M. Karplus, Rev. Mod. Phys. 44, 602 (1972)

${ }^{19}$ C. V. Kraus, M. M. Wolf, J. I. Cirac, and G. Giedke, Phys. Rev. A 79, 012306 (2009).

${ }^{20}$ J. M. Singer, T. Schneider, and P. F. Meier, Eur. Phys. J. B. 7, 37 (1999).

${ }^{21}$ B. Edegger, V. N. Muthukumar, and C. Gros, Adv. Phys. 56, 927 (2007).

${ }^{22}$ D. Hofstadter, Phys. Rev. B 14, 2239 (1976).

${ }^{23}$ J. G. Analytis, S. J. Blundell, and A. Ardavan, Am. J. Phys. 72, 613 (2004). 